\title{
A Brief Survey Paper on Multi-Legged Robots
}

\author{
Mohammad Behmanesh, Ehsan Amiri Tehranizadeh, Mahmud Iwan Solihin \\ Department of Mechatronics Engineering, UCSI University, 56000, Cheras, Malaysia
}

\begin{tabular}{|c|c|}
\hline Article Info & ABSTRACT \\
\hline $\begin{array}{l}\text { Article history: } \\
\text { Received Jun 3, } 2014 \\
\text { Revised Sep 29, } 2014 \\
\text { Accepted Oct 20, } 2014\end{array}$ & $\begin{array}{l}\text { This paper presents a brief survey on multi-legged robots and their } \\
\text { applications in agriculture such as for harvesting. Multi-legged robots } \\
\text { have the benefit of more flexibility and adapt to different rough } \\
\text { terrain in a better way. They also have very important applications in } \\
\text { fulfilling the dangerous tasks such as mine detecting. }\end{array}$ \\
\hline \multicolumn{2}{|l|}{ Keyword: } \\
\hline $\begin{array}{l}\text { Dynamic modelling } \\
\text { Kinematics } \\
\text { Legged robots } \\
\text { Robotics }\end{array}$ & $\begin{array}{r}\text { Copyright (C) } 2015 \text { Institute of Advanced Engineering and Science. } \\
\text { All rights reserved. }\end{array}$ \\
\hline \multicolumn{2}{|l|}{ Corresponding Author: } \\
\hline \multicolumn{2}{|c|}{$\begin{array}{l}\text { Mohammad Behmanesh, } \\
\text { Department of Mechatronics Engineering } \\
\text { UCSI University, } 56000 \\
\text { Cheras, Malaysia } \\
\text { Email: mohammadbehmanesh@gmail.com }\end{array}$} \\
\hline
\end{tabular}

\section{INTRODUCTION}

It is been a long time since the engineers and scientifics have been interested in robotic science. In fact robots have found their applications in different aspects of today's life gradually. Today there is no factory without some kind of robots helping in the product line. This is due to the capabilities of robots that can make the life easier since the robots can accomplish the dangerous or difficult tasks for humankind. From all different types of walking robots, the multi-legged robot is of more interests, since it demonstrates a better movement over rough ground, especially when compared to the wheeled or tracked mobile robots. The multilegged robots show better flexibility and terrain adaptability at the cost of low speed and increased control complexity [1]

Many researches have been done on the robotics issue from different aspects. Here some of the novel understandings on the six legged robots are presented from the kinematics and dynamic modelling views.

\section{KINEMATICS AND DYNAMICS REVIEW}

In a novel study Roy et al [2] estimated the optimal feet forces and joint torques for on-line control of six-legged robot. They have obtained optimal distributions of feet forces and values of joint torques of a six-legged robot on-line. In this study two approaches have been developed:

- Approach I : minimization of the norm of feet forces

- Approach II: minimization of the norm of joint torques.

According to this research, approach II demonstrates better energy efficiency in compare with approach I. This might be because of a better use of friction between tips of the supporting legs and terrain in approach II. Figure 1 displays the distributions of feet forces yielded by Approaches I and II over two 
locomotion cycles. As it can be seen the forces are symmetrical in left and right legs because they are tolerating the same amount of forces when in the support phase.
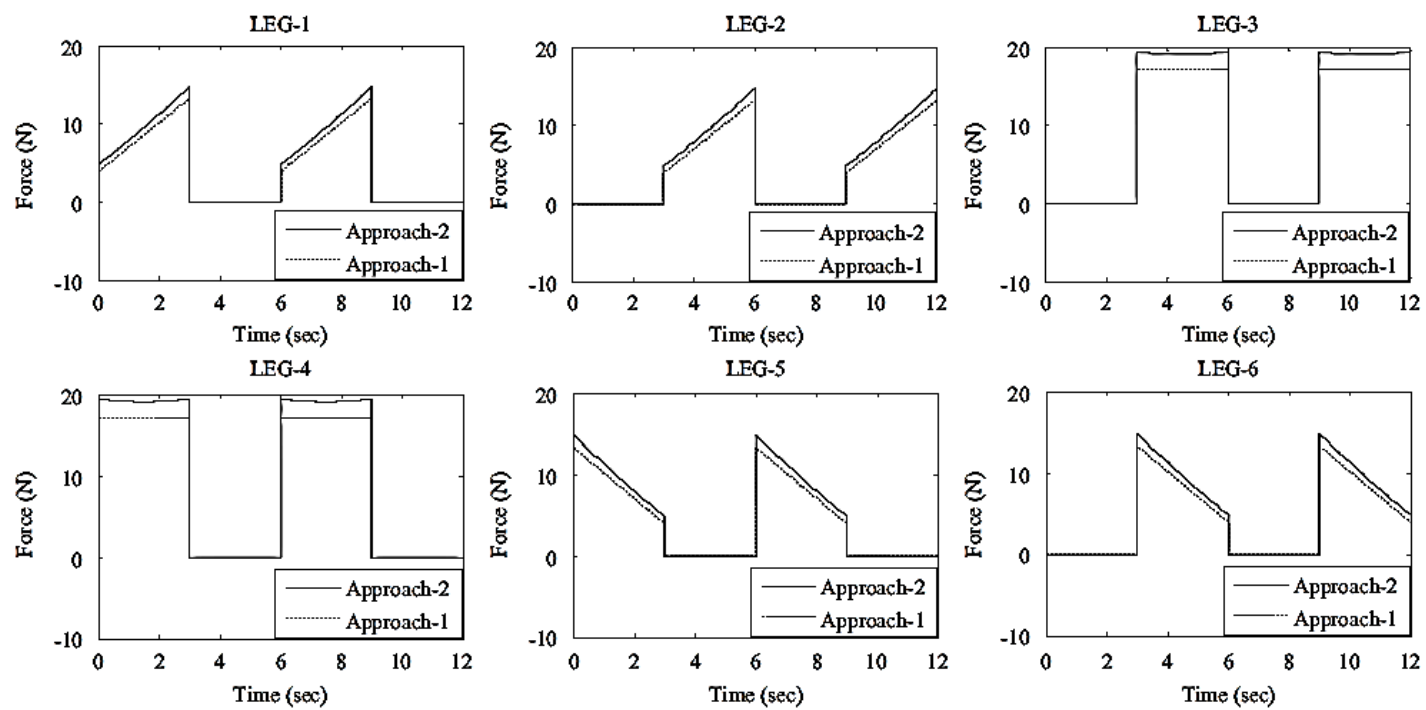

Figure1. Distributions of feet forces obtained by Approaches 1 and 2 (in first phase, the legs: 2, 3 and 6 are on ground, whereas the legs: 1, 4, 5 are on ground in second phase).

Besides the two developed approaches in the previous paper, the energy consumption and stability of the robot have been studied by Roy et al [3]. In this study, the effects of walking parameters, like velocity, stroke and duty factors have been considered. The variations of average power consumption and specific energy consumption with the velocity and stroke are compared for four different duty factors.
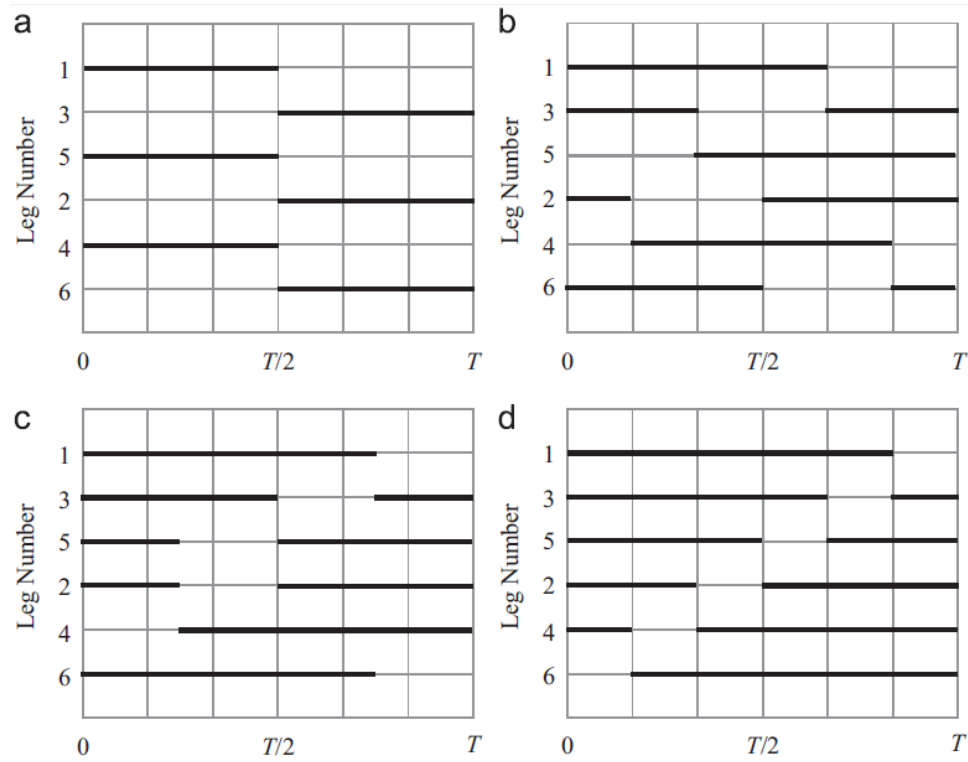

Figure 2. Gait diagrams of the wave gait with duty factors (a) $\beta=1 / 2$, (b) $\beta=2 / 3$, (c) $\beta=3 / 4$ and(d) $\beta=5 / 6$.

Figure 2 shows gait diagrams for different values of duty factors equal to $1 / 2,2 / 3,3 / 4$ and 5/6. It is to be noted that the placement and lifting of a foot that is the period of support phase is shown by the darkened lines in gait diagrams. As it can be shown approach II is more energy efficient in compare with the approach I. Besides, approach II has entrusted less variation in joint torques of the robot compared with the 
approach I. Also, with the increase in duty factor, the maximum values of feet forces and joint torques demonstrate a decrease. In addition, the effect of velocity and stroke on the average power consumption and specific energy consumption has been studied. The results indicate that for all duty factors, average power consumption and specific energy consumption increase with the stroke at a particular velocity. Also, The NESM (Normalized Energy Stability Margin) increases with the increase in duty factor.

In another study, optimal gait for bio-inspired climbing robots using dry adhesion was investigated by Boscariol et al [4]. A quasi-static investigation has been undergone to solve inappropriate redistribution of forces in the preloading of the legs in climbing robots which can cause irreparable detachment of the robot from the vertical surface.

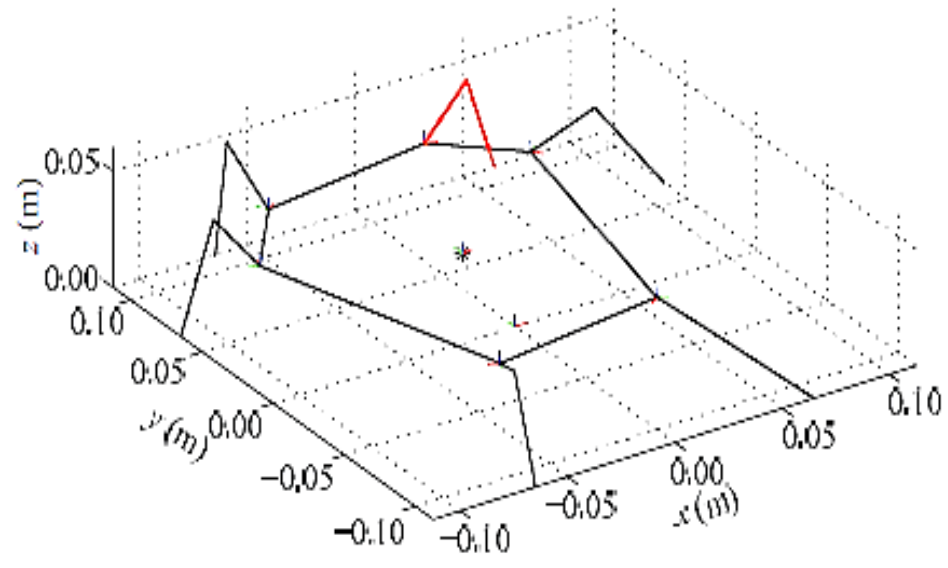

Figure 3. Optimal posture for a vertical wall: leg 1 lifted.

The optimal posture of the robot in a vertical terrain is shown in Figure 3 when only 5 legs are supporting it. The red color leg in Figure 3 is in swing phase. As a result when the center of mass is close to the front legs and the hind legs are extended then the optimal posture is achieved. In addition, the optimal posture has the benefit of a $35 \%$ reduction in total cost.

Also Roy et al [5] analyzed the energy consumption of a six-legged robot during its turning motion over a flat terrain. For this study they considered the gait parameters on energy consumption and stability of the robot. The results indicate that as it was expected increment in angular velocity increases the average power consumption for a particular value of duty factor. Besides, for all duty factors both the specific resistance and the average power consumption increase with angular stroke at a given angular velocity.

Table 1 Average value of the squares of joint torques during turning motion with different duty factors.

\begin{tabular}{ccc}
\hline \multirow{2}{*}{ Duty Factor $(\beta)$} & \multicolumn{2}{c}{$\begin{array}{c}\text { Average of the squares of joint torques } \\
\left(\mathrm{N} . \mathrm{m}^{2}\right)\end{array}$} \\
\cline { 2 - 3 } & Approach I & Approach II \\
\hline $1 / 2$ & 7.2513 & 4.0773 \\
$2 / 3$ & 5.5217 & 2.9939 \\
$3 / 4$ & 4.9860 & 2.6133 \\
$5 / 6$ & 4.5577 & 2.2946
\end{tabular}

Angular stroke $=8$ deg. , Angular velocity $=2$ deg./sec

Height of trunk body $=0.13 \mathrm{~m}$, Turning radius $=1 \mathrm{~m}$

Table 1 shows the average values of the squares of joint torques of the robot for generating waveturning gait patterns with various duty factors, as obtained by approaches I and II. Results indicate that for both approaches the increment in duty factors gives the increment in the average value of the squares of joint torques during one complete locomotion cycle. 
In addition, Roy et al [6] predicted stability margin and energy consumption in turning gaits of sixlegged robots by using soft computing-based expert systems. They developed four different soft computingbased expert systems (that is, Approaches 1 through 4) for predicting specific energy consumption and stability margin for turning motion of a six-legged robot. Approach 1 is back propagation algorithm- turned multiple adaptive neuro-fuzzy inference system, while approach 2 is GA-tuned multiple adaptive neuro-fuzzy inference system then approach 3 is GA-tuned coactive neuro-fuzzy inference system (GACANFIS), finally the approach 4 is GA-tuned back-propagation neural network (GABPNN). By comparing these four approaches, it is observed that approach 2 demonstrates a better accuracy in predictions. This might be because of a GA in place of BP algorithm and using two separate ANFIS structures for the two outputs. For the generalized bell-shaped membership function distributions, the membership values are calculated as follows:

$$
\mu_{i}\left(I_{k}\right)=\frac{1}{1+\left\{\left(\frac{I_{k}-c_{i}}{a_{i}}\right)^{2}\right\}^{b_{i}}}
$$

Where $I_{k}$ is the input, and $a_{i}, b_{i}$ and $c_{i}$ are the parameters of the membership function for ith linguistic term corresponding to an input.

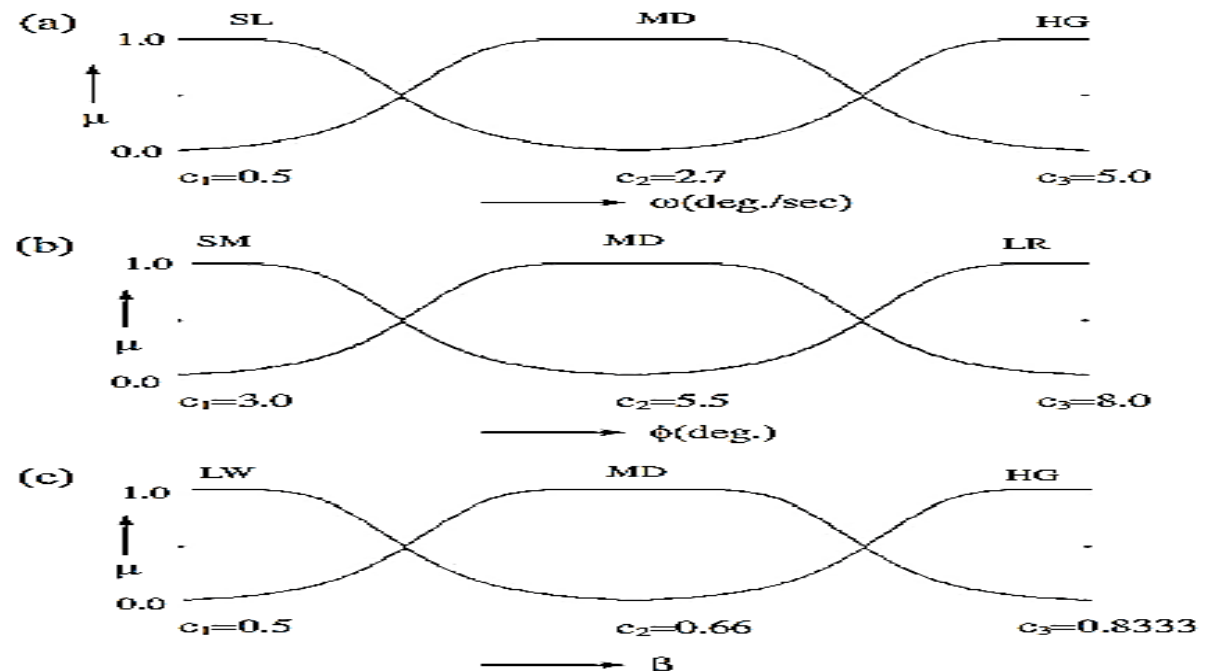

Figure 4. Bell-shaped membership function distributions for the input variables: (a) angular velocity $(\omega)$, (b) angular stroke $(\varphi)$, and (c) duty factor $(\beta)$.

In another study, Wang et al [7] did the mobility analysis on the typical gait of a radial symmetrical six-legged robot. Three types of six-legged robots are studied; one is the insect-wave gait, second is mammal-kick gait and third is insect-mammal mixed gait. It can be observed from the Figure 5 that the mammal-kick gait consumes more energy in compare with the two other gaits. The consumption of energy by the insect-wave gait increases by the increment of turning angle. Also energy consumptions of the dynamics modelling are compared as shown in Figure 6. This theoretical analysis indicates that the energy consumption of the robot in the mammal-kick decreases, in the insect wave gait increases and it stays constant with the insect-mammal mixed gait as a function of increasing the turning angle. 


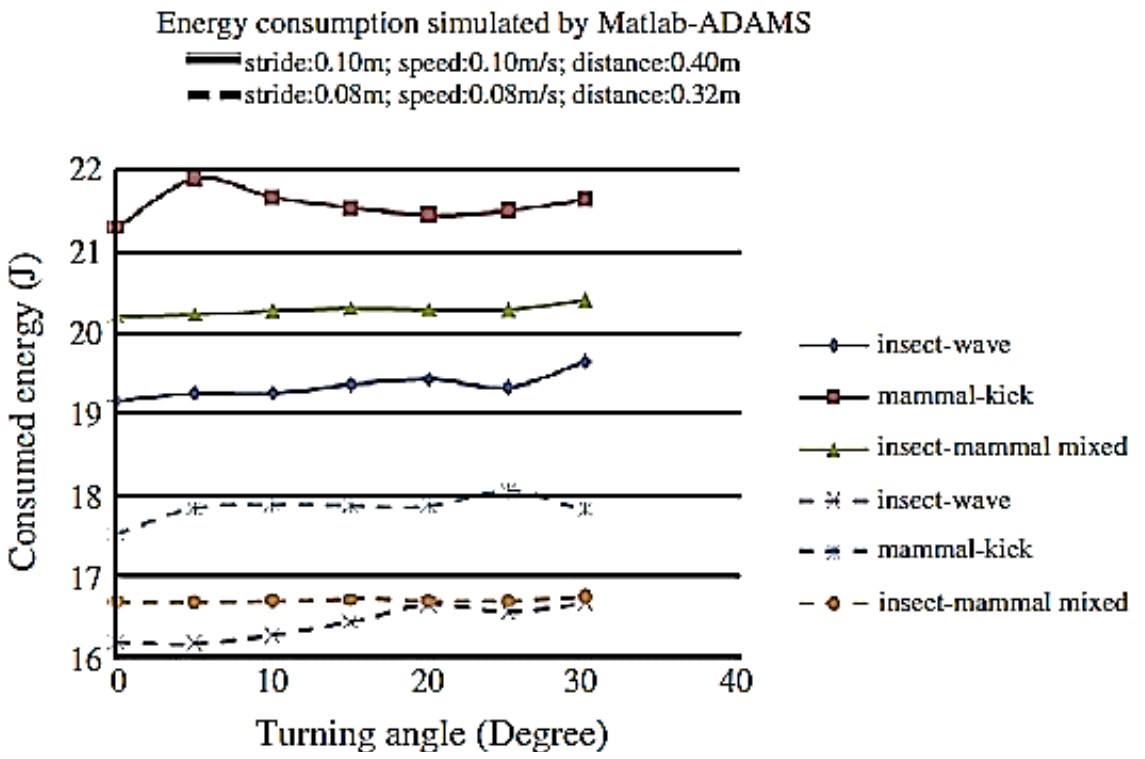

Figure 5. Energy consumption of three gaits by ADAMS simulation (two strides: $0.08 \mathrm{~m}$ and $0.10 \mathrm{~m}$ ).

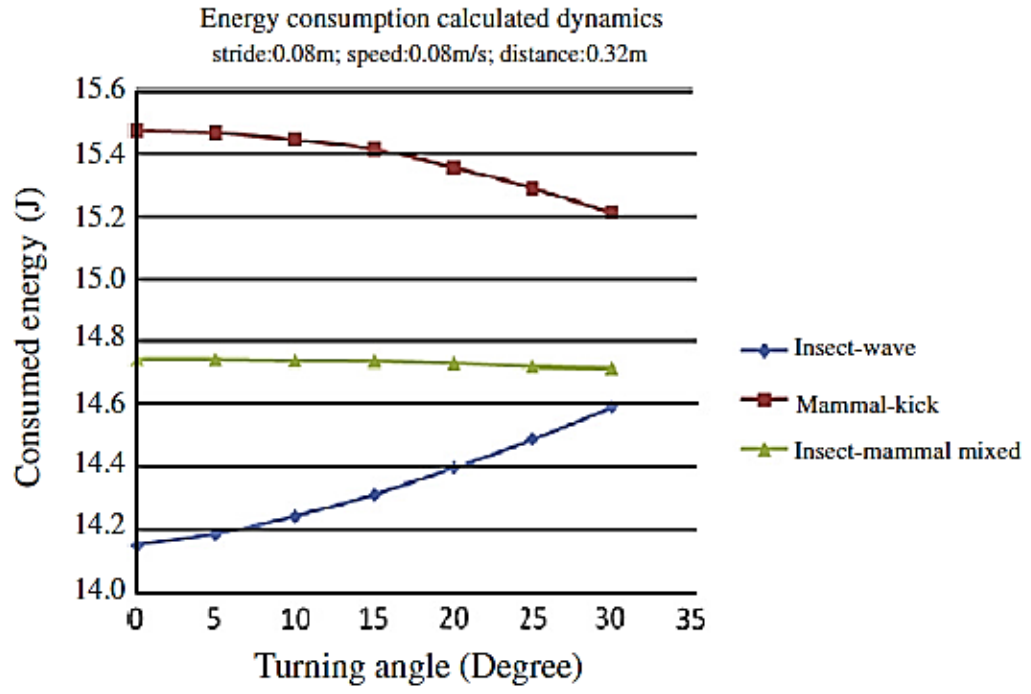

Figure 6. Energy consumption of three gaits calculated through our dynamics model (strides: $0.08 \mathrm{~m}$ ).

In a research by Huang and Nonami [8] high instrumentation technologies for mine detection was developed. Furthermore they studied mine detection strategies using measuring equipment mounted on walking robots based on six-legged teleoperated high technology. Their robot named COMET-I can walk slowly at speed 100-200 m per hour with precise detection mode using six metal detectors. 


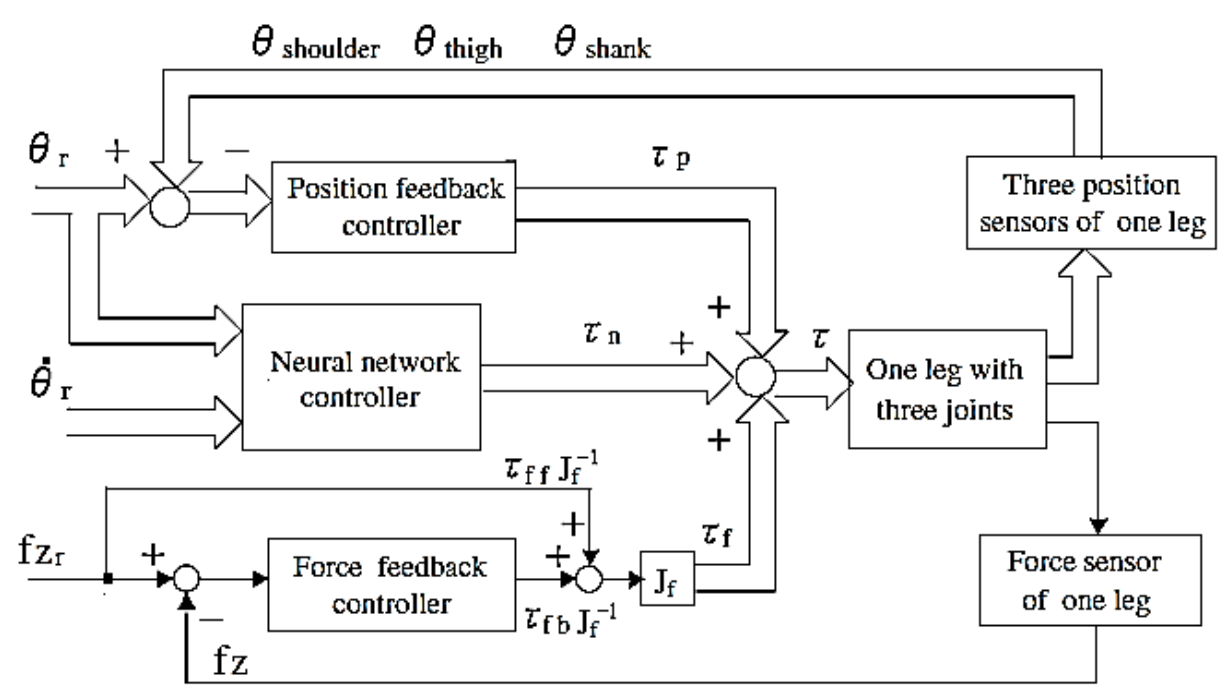

Figure 7. Control system with hybrid position/force neuro controller.

They propose a hybrid neuro control system with position and force control system as shown in Figure 7. In addition, COMET-1 can move $24 \mathrm{~h}$ a day and responses of scanning mock mine using the radar sensor night using IR camera. Therefore, the detected area will increase with using radar sensor for nights and metal detector for days from 2000 to $4000 \mathrm{~m}^{2}$ a day

Mine detection by robots has been of interests for many researchers, such as Gonzalez de Santos et al [9]. They developed a walking robot which carries sensors for detecting and locating the mines in an efficient way. This system benefits from many tools to create a databases of potential alarms and equip the operator with proportional images and graphs by which this system dominates the drawbacks of previous mine detector legged robots, such as weight, speed, omnidirectionality and efficiency.

On the other hand, the robots have applications not only on the ground but also under the sea, Shim et al [10] studied a multi-legged subsea robot system in consideration of mobility and agility. By this research, they successfully derived the dynamic and torque constraint equations. In table 2 the mobility and agility of six-legged robot considering different environment is demonstrated, these data are derived based on the mathematical framework with the dynamic and constraint equations to calculate the acceleration bound of body center.

Table 2. Mobility and agility of six-legged robot considering different environment

\begin{tabular}{cccccc}
\hline \multicolumn{1}{c}{ Class } & \multicolumn{2}{c}{ Ground case } & \multicolumn{2}{c}{ Underwater case } \\
& & Minimum & Maximum & Minimum & Maximum \\
\hline \multirow{2}{*}{ Mobility $\left(\mathrm{m} / \mathrm{s}^{2}\right)$} & $\mathrm{x}$ & -33.96 & 33.96 & -48.78 & 4.78 \\
& $\mathrm{y}$ & -31.04 & 31.04 & -26.7 & 26.7 \\
& $\mathrm{z}$ & -19.62 & 65.46 & -15.62 & 61.35 \\
& $\mathrm{~W}_{\mathrm{x}}$ & -110.4 & 110.4 & -99.25 & 37.24 \\
Agility $\left(\mathrm{rad} / \mathrm{s}^{2}\right)$ & $\mathrm{W}_{\mathrm{y}}$ & -77.2 & 77.2 & -70.78 & 70.78 \\
& $\mathrm{~W}_{\mathrm{z}}$ & -94.59 & 94.59 & -86.04 & 86.04 \\
\hline
\end{tabular}

In a novel study, Pratihar et al [11] established the optimal path with gait generations of a six legged robot by means of a GA-fuzzy approach. For this aim, the six-legged robot should do the following tasks optimally, in the shortest travelling time: first it should move along straight line paths, and then take sharp circular turns and finally cross-ditches. A GA helps the robot to find the proper rules from the different codes, therefore, the robot can find its path and gait simultaneously in an optimal way which means with the maximum average kinematic margin of the ground legged, while minimum number of legged are on the ground and meanwhile the travelling time is minimum as well. 
Besides, Chen and Lan [12] did the simulation and stiffness analysis for hexapod machines. They successfully derived very detailed results which help for calculating the instantaneous stiffness matrix and the deflection of the end-effector of the 6-6 hexapods, for an easy adaption. Furthermore the simulation validates the correction of the mathematics and calculation results.

On the correction capability of a deployed tape-spring hexapod Aridon et al [13] predicted the dynamic behaviour of a tape-spring hexapod using two experimental and numerical approaches. This research determines that an additional DOF by using flexural blades is not necessary due to the flexibility feature of tape-springs.

Furthermore, Yang [14] studied the hexapod robots with a locked joint failure considering the fault tolerance. The failure results in a serious mobility loss. This research indicates that this failure does not affect the stability of a gait however it imposes the workplace of the failed leg. The solution that they have introduced is that the hexapod should discontinue the movement of the body with respect to leg swing while the damaged leg is swung passively by the translation of the body.

Climbing robots have been of a great interest as well as the other types of robots, since they have the capabilities of moving on the large buildings or in the places where is dangerous for human. Therefore a research on legged climbing robot which has the application as maintenance in hazardous environments was done by Luk et al [15]. This robot is named Robug IIs and has insect-like structure which can move in a variety of the terrain. Their method successfully was applied and the results indicate a smooth and accurate movement of the robot, which the GA- fuzzy functions are shown in Figure 8.

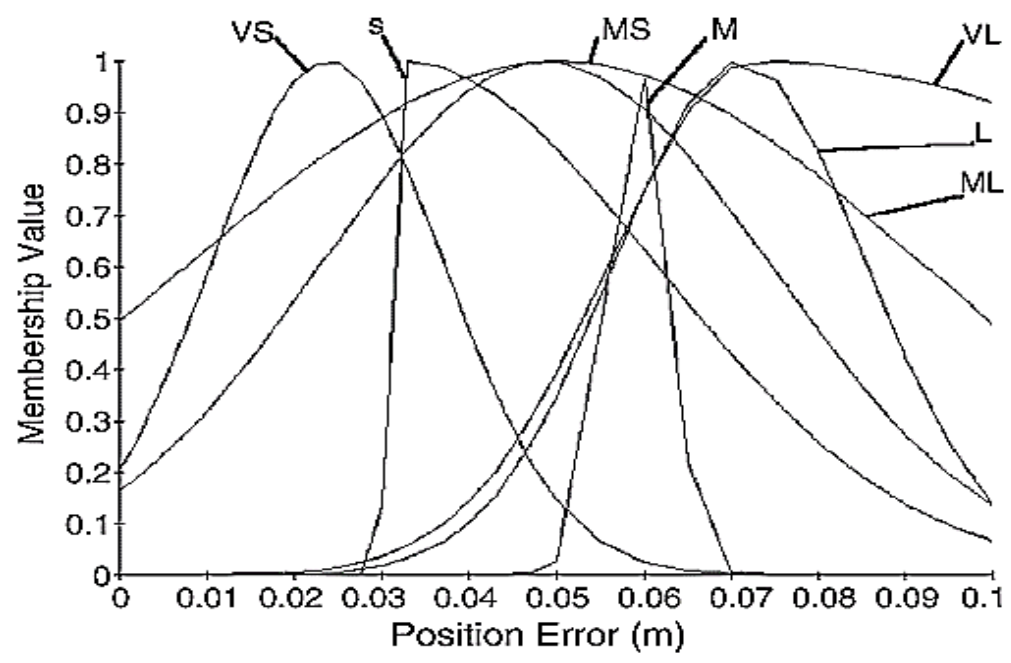

Figure 8. The membership functions after GA optimization.

In another study, for improving the speed control part Yu et al [16] studied the modelling and control of a single-legged robot. They aimed to provide a better method to determine the control parameters. A comparison between the proposed method and the Raibert method showed that the proposed control method is valid and feasible while being easy to achieve and high precision.

In addition Aiyama et al [17], investigated the cooperative transportation by two four-legged robots. Their strategy is that the robots should move autonomously while cooperate to transport an object over a high place or rough terrain. For this aim they adopt a method by which the robots get information only with implicit communication.

In an interesting study, Martín et al [18] combined a fuzzy-Markov method and a population of extended Kalman filters to localize the legged robots. They developed a new approach in case of a strong efficiency requirement which is robot vision-based self-localization in dynamic and noisy environments for legged robots. The results indicate that this new approach is suitable for the RoboCup conditions. This new approach benefits from robust in noisy environments, converging from scratch and recovering from kidnapping.

Meanwhile, Anshar and Williams [19] studied another approach that is fast learn-to-walk for four legged robots. This approach aims to decrease the wear and tear of robot motors, joints and other hardware. They run several tests based on the standard GA and the results can be seen in Figure 9. 


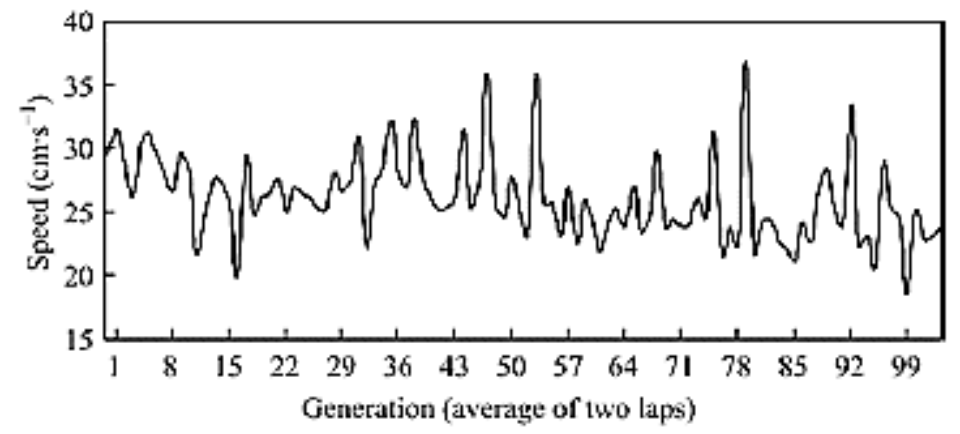

Figure 9. Speed vs generation based on standard GA.

Their research determines that the extended GA is performing better than fitness-driven search and standard GA, because in extended GA the incorrect parameters can be prevented.

Also, Kumar K et al[20] used MATLAB and Simulink to do dynamic modelling and simulation of a four-legged jumping robot with compatible legs. According to their simulation, the robot can run at a speed of $1.5 \mathrm{~m} / \mathrm{s}$ approximately, furthermore their simulation was animated to give a clear understanding of the running cycle. A PD control algorithm was implemented to the simulated model to control the forward speed.

Since it is very important to know the exact location of a robot especially in tasks such as mine detecting, Cobano et al [21] developed DGPS (Differential Global Positioning System) antenna and the DGPS receiver system for SILO4 (Figure 10) legged robot.
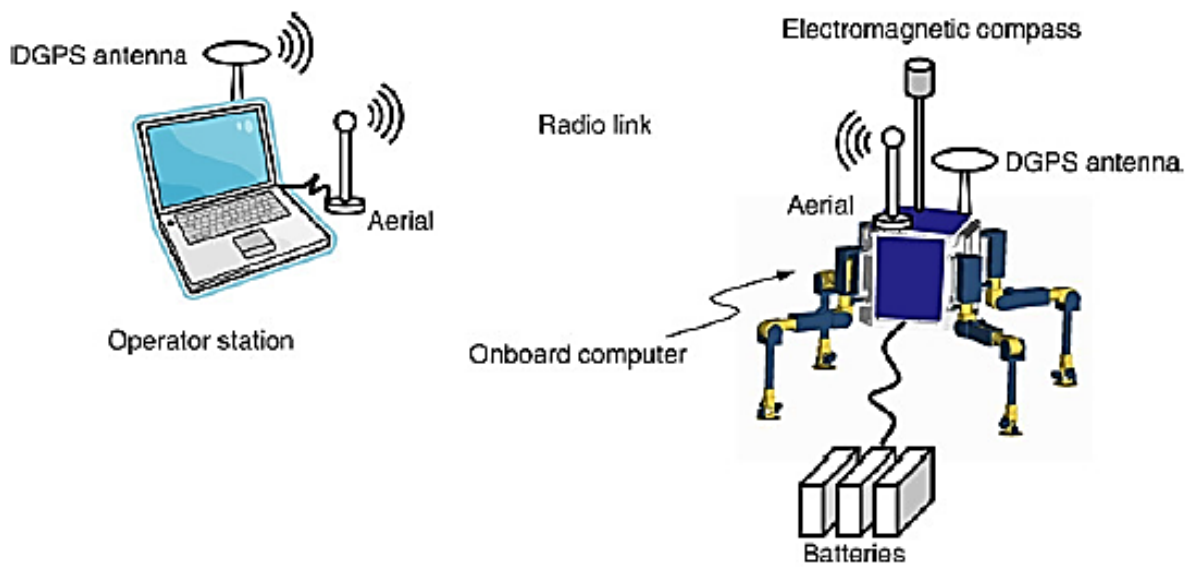

Figure 10. The SILO4 system.

The experiments showed that the data acquired of the electromagnetic compass in concern with the odometry data can achieve the adequate assessment of the robot's position. This paper determines that the position of the robot in outdoor environment can be located with an accuracy of about $\pm 20 \mathrm{~mm}$.

In addition, the robots have found their application in the agricultural industry, such as harvesting. Design and control of an apple harvesting robot was studied by De-An et al [22]. They successfully designed a robot (Figure 11) which has the capabilities to detect, locate and pick the fruit with no damage on the fruit or the tree. For detection and localizing the fruit, a vision-based module is used by the robot, and for approaching and picking the fruits, a control system consisted of manipulator and the end-effector is used. 


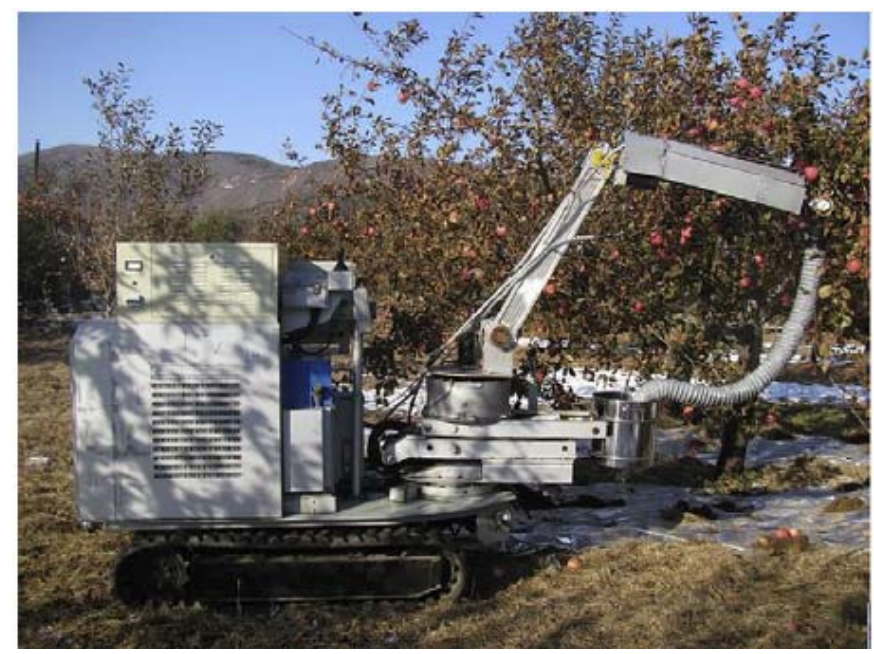

Figure 11. Harvesting experiments in an orchard.

Also, Ji et al [23] investigated a guide system for apple harvesting by means of and automatic vision system. Their simulation on Visual C++ indicates that using SVM (Support Vector Machine) for apple harvesting results in a higher recognition rate than using only the shape or color characteristics of the fruit, besides the harvesting time in this method is shorter.

Furthermore, the robots have application in the harvesting not only for the tree fruits but also for the field fruits such as strawberry. Hayashi et al [24] evaluated a robot for harvesting strawberry in a field. The robot was successfully designed with the abilities to operate at nights, handle peduncle and sharing tasks with human workers.

In addition, Rath and Kawollek [25] investigated a Gerbera Jamesonii (kind of flower) harvesting robot. This robot detects the pedicels by images of one camera, then a three-dimensional modeling of cutflower pedicels are performed, by means of using the second camera images. Analyzing both images from camera enables the automatic harvesting by almost $97 \%$ of correct harvesting.

A survey of design aspects and technologies for climbing robots was undergone by Schmidt and Berns [26] since it is still an unsolved problem. They examined the applications and requirements for robot locomotion as well as for attraction to the vertical structures are discussed. Their results indicate that so far there is no system which could meet the given requirements. Only for a specific setup or certain environments there are special solutions for robotic prototypes.

In a wonderful study, Zhang et al 2014 [27] developed a bionic hexapod robot for walking on unstructured terrain. In this paper, they explained in details the designed methodology and control system of the aforementioned robot, HITCR-II. The Figure12 shows the structure of the robot trunk.

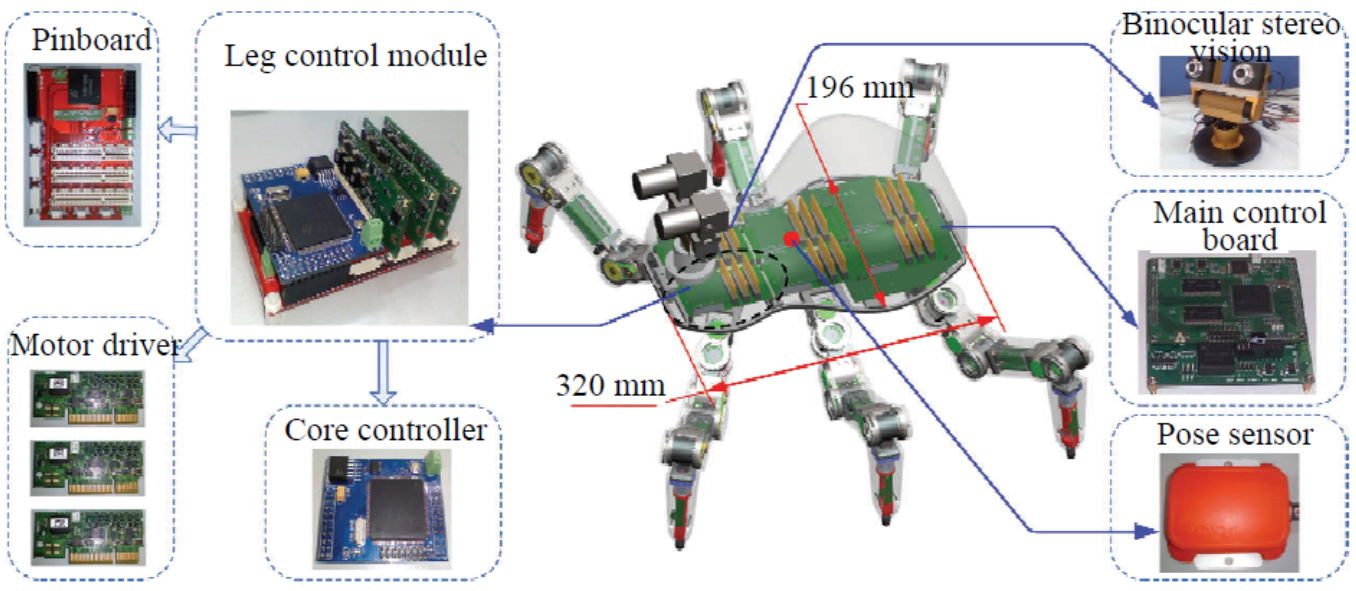

Figure 12. The structure of HITCR-II trunk 
They optimized the structure parameters, then analyzed the relationship between dexterity and stability margin. The simulation and experiment results indicate that the robot is capable of walking on the unstructured terrain.

Recently the number of papers for designing a robot has increased, for instance $\mathrm{Pa}$ and $\mathrm{Wu}$ [28] have published their novel design experiment of a hexapod robot with a servo control and a man-machine interface. As shown in Figure 13. The gait sequences of the robot were well designed. Their experiment determines that the hexapod robot is capable of walking and the highly sensitive detection of obstacles.

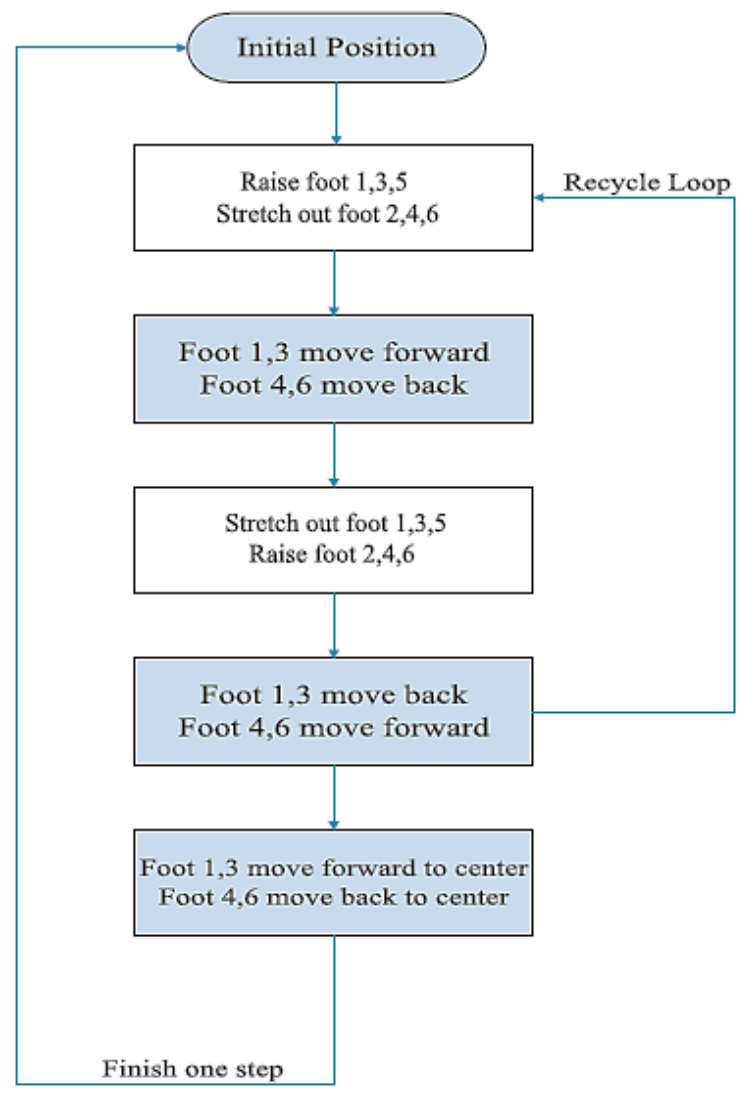

Figure 13 Gait sequence of the hexapod robot.

While in another study, an underwater hexapod robot AQUA was simulated by Georgiades et al 2009 [29]. The motion of the robot based on its paddle oscillation was simulated. Then the simulation results were applied on the experimental robot, the results were to improve the movement of the robot underwater by having a better force and torque distribution according to the paddle oscillation.

Also, dynamic analysis of a hexapod robot was carried out using the concept of multibody dynamics by Mahapatra et al [30]. They benefited from CAD, ADAMS and CATIA softwares to do the analysis. The results indicate that retraction phase requires much higher torque than protraction phase for all the joints. It is because of the weight of the trunk body carried out by the legs.

In addition, the reconfiguration of the six-legged robot is optimized by using hexa-quad transformation [31]. They proposed this method for a certain situations that a leg can do the other legs tasks and some legs are disabled. They successfully simulated in real time and verified the result numerically.

\section{CONCLUSION}

In this paper, a short review on multi-legged robots as well as some applications has been done. It has been understood that the legged robot have so much benefits and more flexibility compared to other types of robots. They also are capable of moving in rough terrain. Furthermore, for risky and dangerous tasks such as mine detection, or for tall buildings and underwater tasks they are very good options. In some research it 
was determined that the position of the robot in outdoor environment can be located with an accuracy of about $\pm 20 \mathrm{~mm}$. Also some solutions were given in some papers for the locked joint failure.

\section{REFERENCES}

[1] S.M. Song and K.J.Waldron, "Machines That Walk: The Adaptive Suspension Vehicle", The MIT Press, Cambridge, Massachusetts, 1989.W. K. Chen, Linear Networks and Systems (Book style). Belmont, CA: Wadsworth, pp. 123135, 1993.

[2] Shibendu Shekhar Roy, Ajay Kumar Singh, Dilip Kumar Pratihar, "Estimation of optimal feet forces and joint torques for on-line control of six-legged robot,” Robotics and Computer-Integrated Manufacturing, Volume 27, Issue 5, October 2011, Pages 910-917, ISSN 0736-5845, 10.1016/j.rcim.2011.03.002.

[3] Shibendu Shekhar Roy, Dilip Kumar Pratihar, "Dynamic modeling, stability and energy consumption analysis of a realistic six-legged walking robot,” Robotics and Computer-Integrated Manufacturing, Volume 29, Issue 2, April 2013, Pages 400-416, ISSN 0736-5845, 10.1016/j.rcim.2012.09.010.

[4] Paolo Boscariol, Michael A. Henrey, Yasong Li, Carlo Menon, "Optimal Gait for Bioinspired Climbing Robots Using Dry Adhesion: A Quasi-Static Investigation,” Journal of Bionic Engineering, Volume 10, Issue 1, January 2013, Pages 1-11, ISSN 1672-6529, 10.1016/S1672-6529(13)60193-6.

[5] Shibendu Shekhar Roy, Dilip Kumar Pratihar, "Effects of turning gait parameters on energy consumption and stability of a six-legged walking robot,” Robotics and Autonomous Systems, Volume 60, Issue 1, January 2012, Pages 72-82, ISSN 0921-8890, 10.1016/j.robot.2011.08.013.

[6] Shibendu Shekhar Roy, Dilip Kumar Pratihar, "Soft computing-based expert systems to predict energy consumption and stability margin in turning gaits of six-legged robots," Expert Systems with Applications, Volume 39, Issue 5, April 2012, Pages 5460-5469, ISSN 0957-4174, 10.1016/j.eswa.2011.11.039.

[7] Zhiying Wang, Xilun Ding, Alberto Rovetta, Alessandro Giusti, "Mobility analysis of the typical gait of a radial symmetrical six-legged robot,” Mechatronics, Volume 21, Issue 7, October 2011, Pages 1133-1146, ISSN 09574158, 10.1016/j.mechatronics.2011.05.009.

[8] Qing-Jiu Huang, Kenzo Nonami, "Humanitarian mine detecting six-legged walking robot and hybrid neuro walking control with position/force control,” Mechatronics, Volume 13, Issues 8-9, October 2003, Pages 773-790, ISSN 0957-4158, 10.1016/S0957-4158(03)00002-3.

[9] P. Gonzalez de Santos, J.A. Cobano, E. Garcia, J. Estremera, M.A. Armada, “A six-legged robot-based system for humanitarian demining missions,” Mechatronics, Volume 17, Issue 8, October 2007, Pages 417-430, ISSN 09574158, 10.1016/j.mechatronics.2007.04.014.

[10] Hyungwon Shim, Bong-Huan Jun, Pan-Mook Lee, "Mobility and agility analysis of a multi-legged subsea robot system," Ocean Engineering, Volume 61, 15 March 2013, Pages 88-96, ISSN 0029-8018, 10.1016/j.oceaneng.2013.01.001.

[11] Dilip Kumar Pratihar, Kalyanmoy Deb, Amitabha Ghosh, Optimal path and gait generations simultaneously of a six-legged robot using a GA-fuzzy approach, Robotics and Autonomous Systems, Volume 41, Issue 1, 31 October 2002, Pages 1-20, ISSN 0921-8890,

[12] J. Chen, F. Lan, "Instantaneous stiffness analysis and simulation for hexapod machines,” Simulation Modelling Practice and Theory, Volume 16, Issue 4, April 2008, Pages 419-428, ISSN 1569-190X

[13] G. Aridon, L. Blanchard, A. Allezy, D. Rémond, R. Dufour, "On the correction capability of a deployed tape-spring hexapod," Mechanism and Machine Theory, Volume 43, Issue 8, August 2008, Pages 1009-1023, ISSN 0094-114X

[14] Jung-Min Yang, "Tripod gaits for fault tolerance of hexapod walking machines with a locked joint failure," Robotics and Autonomous Systems, Volume 52, Issues 2-3, 31 August 2005, Pages 180-189, ISSN 0921-8890,

[15] Bing L. Luk, David S. Cooke, Stuart Galt, Arthur A. Collie, Sheng Chen, "Intelligent legged climbing service robot for remote maintenance applications in hazardous environments," Robotics and Autonomous Systems, Volume 53, Issue 2, 30 November 2005, Pages 142-152, ISSN 0921-8890

[16] Xiaowen Yu, Chenglong Fu, Ken Chen, "Modeling and Control of a Single-legged Robot,” Procedia Engineering, Volume 24, 2011, Pages 788-792, ISSN 1877-7058,

[17] Y Aiyama, M Hara, T Yabuki, J Ota, T Arai, "Cooperative transportation by two four-legged robots with implicit communication,” Robotics and Autonomous Systems, Volume 29, Issue 1, 31 October 1999, Pages 13-19, ISSN 0921-8890,

[18] Francisco Martín, Vicente Matellán, Pablo Barrera, José M. Cañas, "Localization of legged robots combining a fuzzy-Markov method and a population of extended Kalman filters," Robotics and Autonomous Systems, Volume 55, Issue 12, 31 December 2007, Pages 870-880, ISSN 0921-8890, 10.1016/j.robot.2007.09.006.

[19] Muh. Anshar, Mary-Anne Williams, "Extended Evolutionary Fast Learn-to-Walk Approach for Four-Legged Robots,” Journal of Bionic Engineering, Volume 4, Issue 4, December 2007, Pages 255-263, ISSN 1672-6529

[20] Ganesh Kumar K., Pushparaj Mani Pathak, "Dynamic modelling \&amp; simulation of a four legged jumping robot with compliant legs,” Robotics and Autonomous Systems, Volume 61, Issue 3, March 2013, Pages 221-228, ISSN 0921-8890

[21] J.A. Cobano, J. Estremera, P. Gonzalez de Santos, "Location of legged robots in outdoor environments," Robotics and Autonomous Systems, Volume 56, Issue 9, 30 September 2008, Pages 751-761, ISSN 0921-8890

[22] Zhao De-An, Lv Jidong, Ji Wei, Zhang Ying, Chen Yu, "Design and control of an apple harvesting robot," Biosystems Engineering, Volume 110, Issue 2, October 2011, Pages 112-122, ISSN 1537-5110 
[23] Wei Ji, Dean Zhao, Fengyi Cheng, Bo Xu, Ying Zhang, Jinjing Wang, “Automatic recognition vision system guided for apple harvesting robot,” Computers \& Electrical Engineering, Volume 38, Issue 5, September 2012, Pages 1186-1195, ISSN 0045-7906

[24] Shigehiko Hayashi, Kenta Shigematsu, Satoshi Yamamoto, Ken Kobayashi, Yasushi Kohno, Junzo Kamata, Mitsutaka Kurita, "Evaluation of a strawberry-harvesting robot in a field test,” Biosystems Engineering, Volume 105, Issue 2, February 2010, Pages 160-171, ISSN 1537-5110

[25] Thomas Rath, Marco Kawollek, "Robotic harvesting of Gerbera Jamesonii based on detection and threedimensional modeling of cut flower pedicels," Computers and Electronics in Agriculture, Volume 66, Issue 1, April 2009, Pages 85-92, ISSN 0168-1699

[26] Daniel Schmidt, Karsten Berns, Climbing robots for maintenance and inspections of vertical structures-A survey of design aspects and technologies, Robotics and Autonomous Systems, Volume 61, Issue 12, December 2013, Pages 1288-1305, ISSN 0921-8890

[27] He Zhang, Yubin Liu, Jie Zhao, Jie Chen, Jihong Yan, Development of a bionic hexapod robot for walking on unstructured terrain, Journal of Bionic Engineering, Volume 11, Issue 2, April 2014, Pages 176-187, ISSN 1672652

[28] P.S. Pa, C.M. Wu, Design of a hexapod robot with a servo control and a man-machine interface, Robotics and Computer-Integrated Manufacturing, Volume 28, Issue 3, June 2012, Pages 351-358, ISSN 0736-5845

[29] Christina Georgiades, Meyer Nahon, Martin Buehler, Simulation of an underwater hexapod robot, Ocean Engineering, Volume 36, Issue 1, January 2009, Pages 39-47, ISSN 0029-8018

[30] Abhijit Mahapatra, Shibendu Shekhar Roy, Dilip Kumar Pratihar, Modeling and Simulation of Wave Gait of a Hexapod Walking Robot: A CAD/CAE Approach, International Journal of Robotics and Automation (IJRA) Vol. 2, No. 3, September 2013, pp. 104 111,ISSN: 2089-4856

[31] Addie Irawan, Yee Yin Tan, Optimizing Hexapod Robot Reconfiguration using Hexa-Quad Transformation, International Journal of Robotics and Automation (IJRA) Vol. 3, No. 2, June 2014, pp. 139 150,ISSN: 2089-4856

\section{BIOGRAPHIES OF AUTHORS}

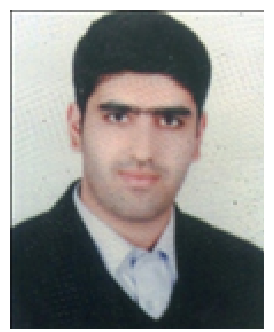

Mohammad Behmanesh is a researcher at Mechatronics Engineering at UCSI University in Malaysia. Currently he is doing a novel research on robotics to develop a six-legged robot used for planting. He has done some other research on bio-composite technology as well and he has one journal paper.

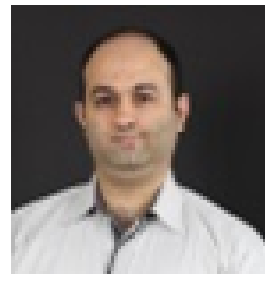

Ehsan Amiri Tehranizadeh has been serving UCSI University, Malaysia as a lecturer in Mechatronics Engineering since 2009. He received his BSc. in Mechanical Engineering from Kerman University, Iran and MSc in Biomedical Engineering from Sharif University of Technology, Iran and is awarded a Chartered Engineer status from UK Engineering Council due to his extensive experiences (around 13 years) in Mechanical and Mechatronics Engineering including 7 years at Aircraft Manufacturing Industries. His research interests include PEM Fuel Cells, Sustainable Energies, Robotics and Automation

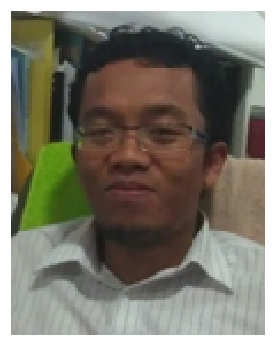

Mahmud Iwan Solihin is currently an Assistant Professor in Mechatronics Engineering at the UCSI University, Malaysia. He received his BS in Electrical Engineering from the Gadjah Mada University, Indonesia in 2001. He specialized his MSc in Mechatronics Engineering at IIUM, Malaysia and received his PhD from the university in 2012. His research interests include intelligent systems, robust control, optimization, robotics and automation. He has published more than 30 international journals, conference papers and book chapters. 